Workshop on high-energy photon collisions at LHC, 2008 CERN, April 22-25

\title{
Factorisation breaking in diffraction
}

\author{
Alessia Bruni, INFN Bologna \\ on behalf of the $\mathrm{H} 1$ and ZEUS collaborations
}

\section{OUTLINE}

- Diffractive hard selection

- Hard diffraction in QCD

- Diffractive Parton Density Functions

- Diffractive final states (open charm, jets)

- Events with leading neutrons 


\section{Diffractive event selection}

\section{HERA: $27.5 \mathrm{GeV} e+920 \mathrm{GeV} p, \sqrt{s}=318 \mathrm{GeV}$}

A large fraction (10\% - 20\%) of DIS events is diffractive
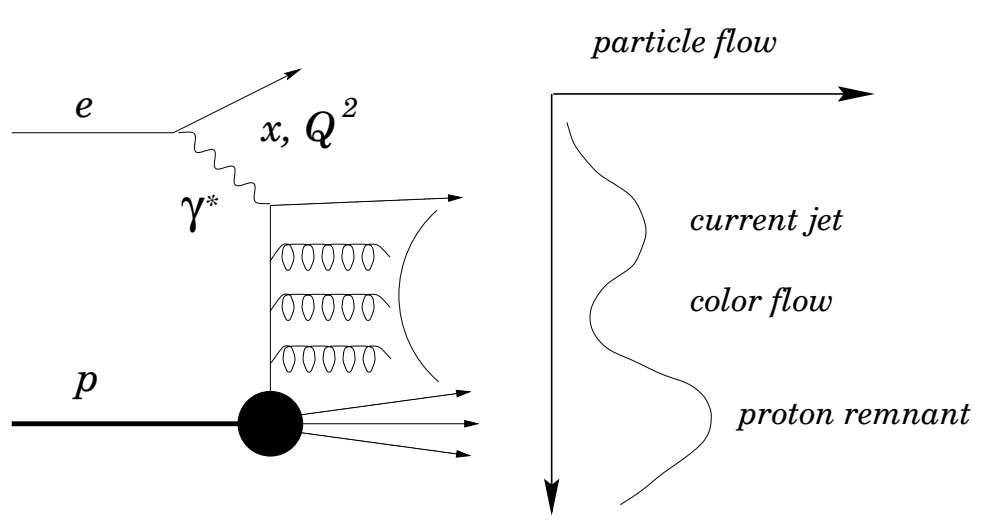

DIS

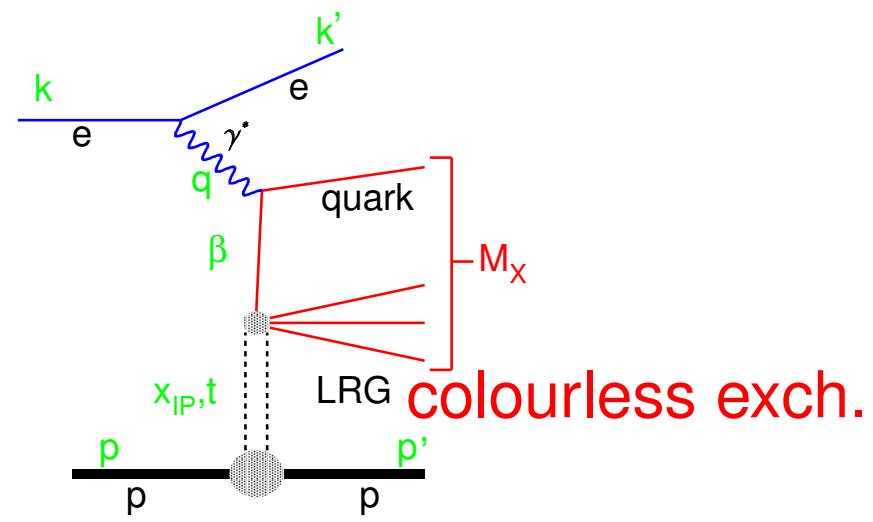

DIS -Diffraction

- Beam particles emerge intact or dissociated into low-mass states; energy $\simeq$ beam energy (within a few \%)

- Final-state particles separated by Large Rapidity Gap (LRG)

- Interaction mediated by t-channel exchange of object with vacuum quantum numbers: colour siglet exchange or Pomeron 


\section{Hard diffraction in QCD}

Diffractive processes with one or several large scales are called hard Hard diffraction is the interplay of systems with small size configurations (quark and gluon) and large size configurations (set by the size of the protons)

Application of pQCD relies on the ability to separate small (hard) from large configurations (soft)

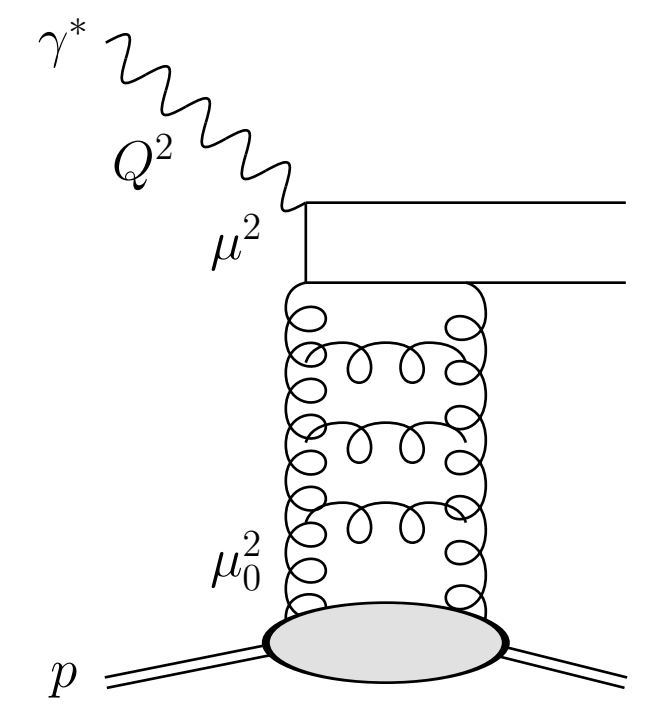

Hard diffraction is a laboratory for QCD studies:

- scale transition soft-hard

- factorisation tests

- dynamics of hard processes

\section{2-gluon diagram}




\section{Kinematic variables}

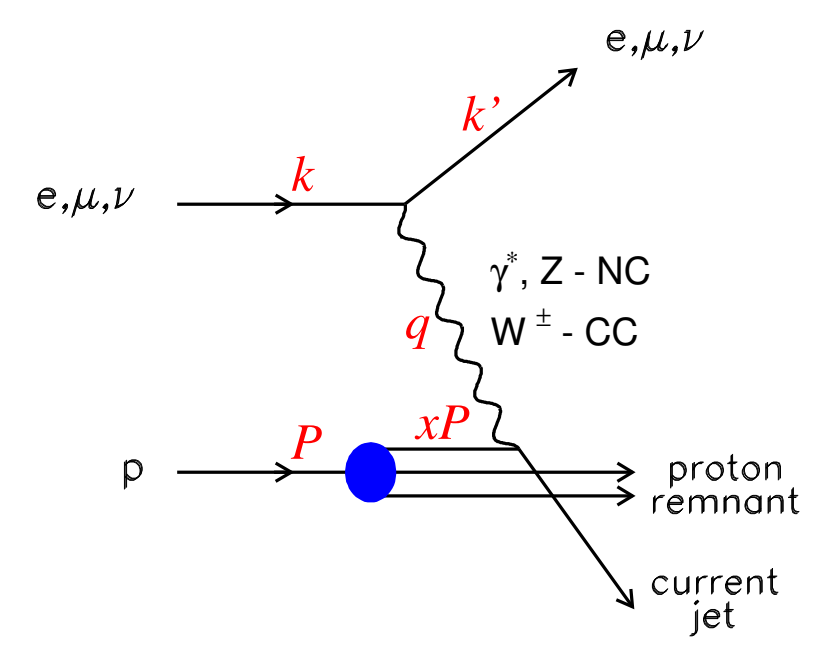

\section{DIS}

$Q^{2}=-q^{2}$, virtuality of exchanged boson

$x_{B}=Q^{2} / 2 p \cdot q$, Bjorken variable

fraction of proton's momentum carried by struck quark

$y \quad=Q^{2} / s x, \gamma$ inelasticity

$W \quad$ photon-proton centre of mass energy

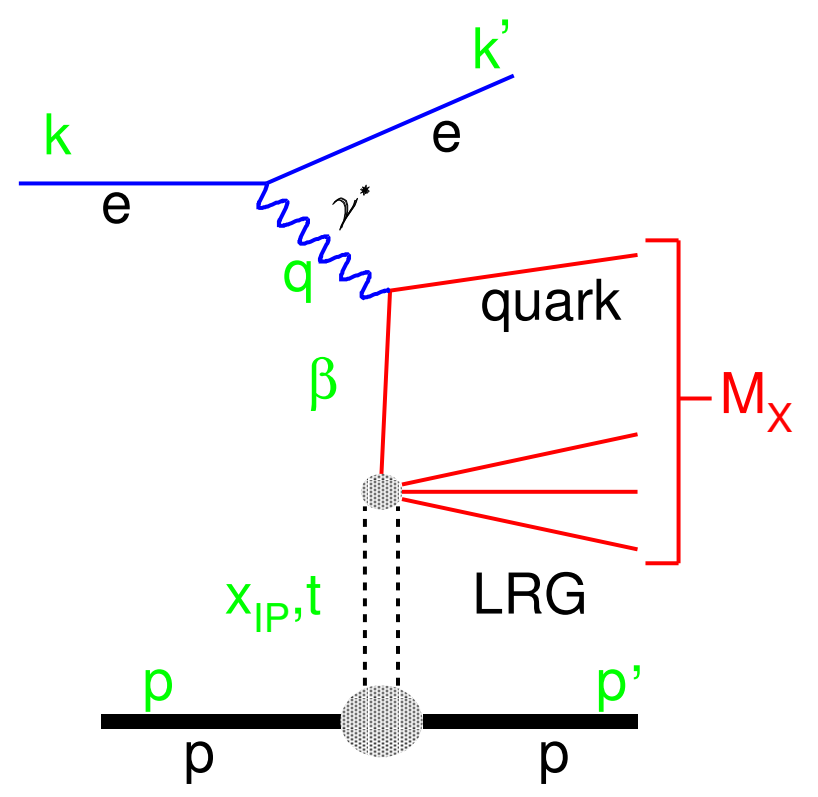

\section{Diffractive DIS}

$x_{\mathbb{P}}$ fraction of proton's momentum of colour singlet exchange $=\frac{q \cdot\left(p-p^{\prime}\right)}{q \cdot p} \simeq \frac{Q^{2}+M_{X}^{2}}{Q^{2}+W^{2}}$

$\beta \quad$ fraction of Pomeron's momentum carried by quark coupling to $\gamma^{*}=\frac{Q^{2}}{2 q \cdot\left(p-p^{\prime}\right)} \simeq \frac{Q^{2}}{Q^{2}+M_{X}^{2}}=x_{B} / x_{\mathbb{P}}$

$t \quad=\left(p-p^{\prime}\right)^{2}, 4$-momentum transfer squared at $p$-vertex 


\section{QCD factorisation theorem}

\section{Breit rest frame (fast proton)}

DIS

$$
F_{2}\left(x, Q^{2}\right)=\sum_{i=q, g} \int d z \quad f_{i}\left(z, Q^{2}\right) \quad C_{i}\left(x, Q^{2}\right)
$$

structure parton distribution function hard scattering coefficients

functions (universal, dglap) (pqcd)

at LO, coefficients $C_{q}(x)=e_{q}^{2} \delta(1-x), C_{g}(x)=0$

Diffractive DIS

$$
\begin{aligned}
& F_{2}^{D(4)}=\frac{d^{2} F_{2}^{D}\left(x_{\mathbb{P}}, t, x, Q^{2}\right)}{d x_{\mathbb{P}} d t}=\sum_{i=q, g} \int d z \quad \frac{d^{2} f_{i / p}^{D}\left(x_{\mathbb{P}}, t, z, \mu_{F}^{2}\right)}{d x_{\mathbb{P}} d t} \quad C_{i}\left(\frac{x}{z}, \frac{Q^{2}}{\mu_{F}^{2}}\right) \\
& \text { Diffractive PDF same as } F_{2}
\end{aligned}
$$

- Factorisation proved for DDIS and exclusive hard diffraction for large $\mu_{F}$ (Collins, Berera \&Soper, Trentadue \& Veneziano)

- Not true for diffr. hadron-hadron collisions 


\section{Factorisation not expected to hold for $p p$}

- Violation of factorization understood in terms of (soft) rescattering between the 2 hadrons and their remnants, in initial and final state, suppressing the large rapidity gap

(a)

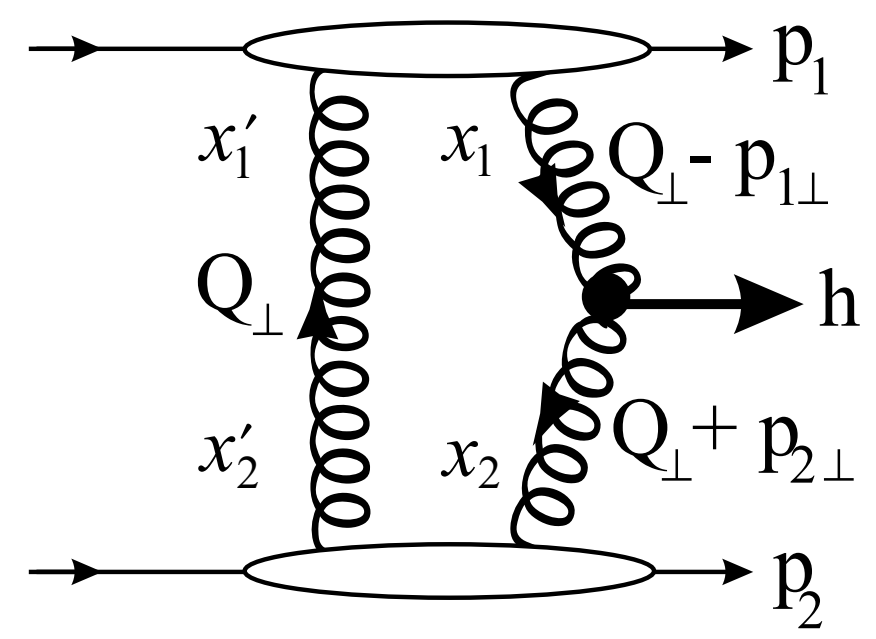

(b)

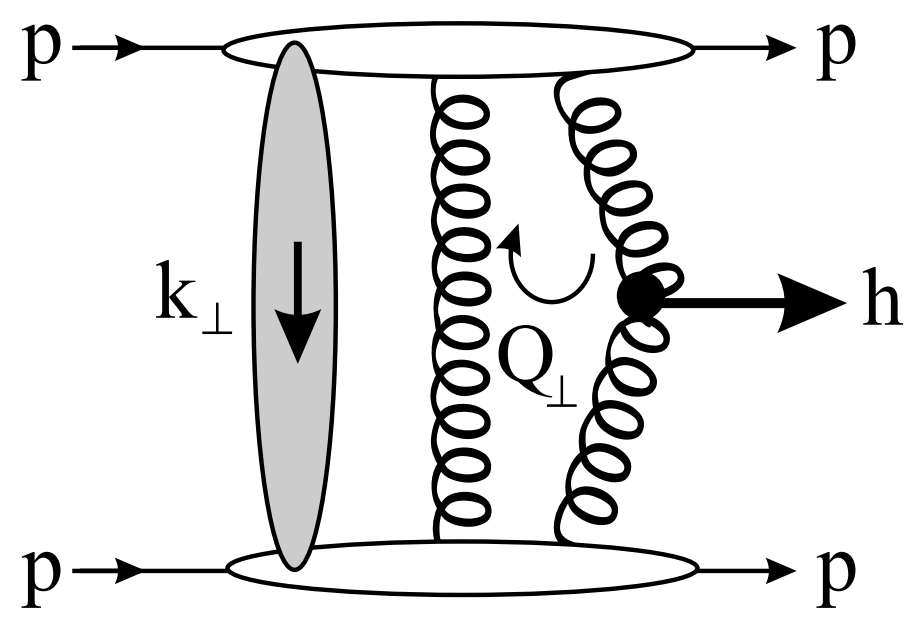

- Suppression observed for diffractive dijet production at Tevatron

- Interest for LHC diffractive production 


\section{Factorisation tests in $\gamma^{*} p$ and $\gamma p$}

\section{Real photon $\left(Q^{2} \simeq 0\right)$ can develop hadronic structure $\Rightarrow$ study fact. breaking at HERA}

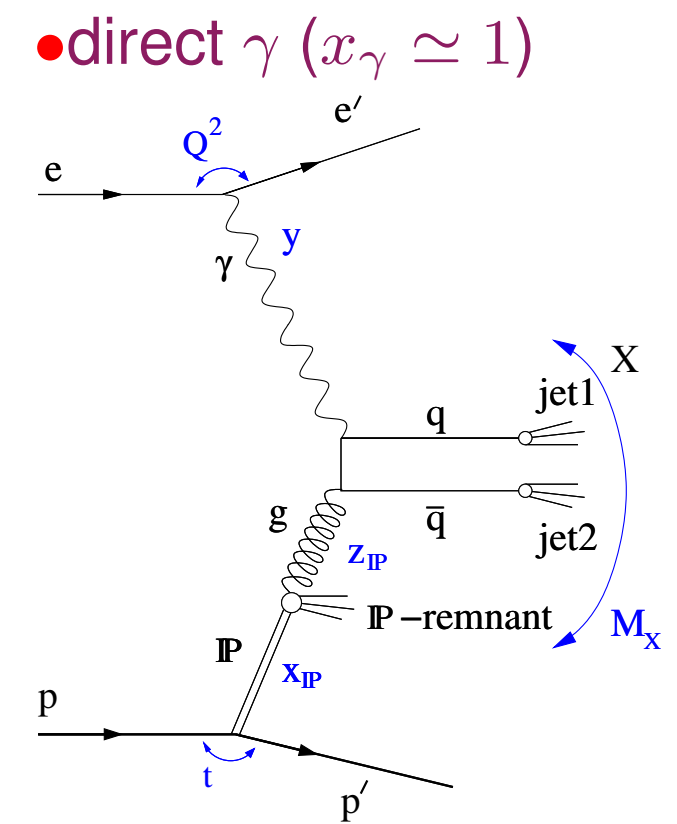

small $\gamma$ couples directly to parton, dominant contribution in DIS and for high $E_{T}$ jets -resolved $\gamma$ (hadron like, at low $x_{\gamma}$ )

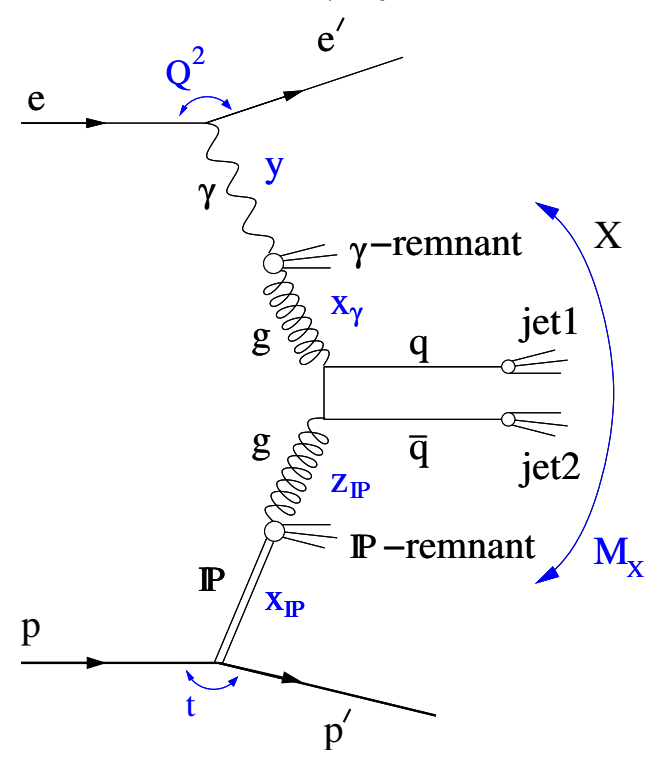

large $\gamma$, soft rescattering expected, dominant contribution for low $E_{T}$ jets

- Theory: direct and resolved processes well separated only at LO

- Experimentally: $x_{\gamma}$ reconstructed by jets, $x_{\gamma}=x_{\gamma}^{O B S}=\frac{\sum\left(E-p_{z}\right)_{j e t s}}{\sum\left(E-p_{z}\right)_{\text {hadrons }}}$ 


\section{Diffractive final states, factorisation tests}

- Basic strategy

- measure $F_{2}^{D}$ from inclusive measurement,

- extract diffractive PDFs from NLO DGLAP fit

- measure an exclusive diffractive final state, open charm and dijets, in DIS and PHP

- compare the measurement to NLO calculation 


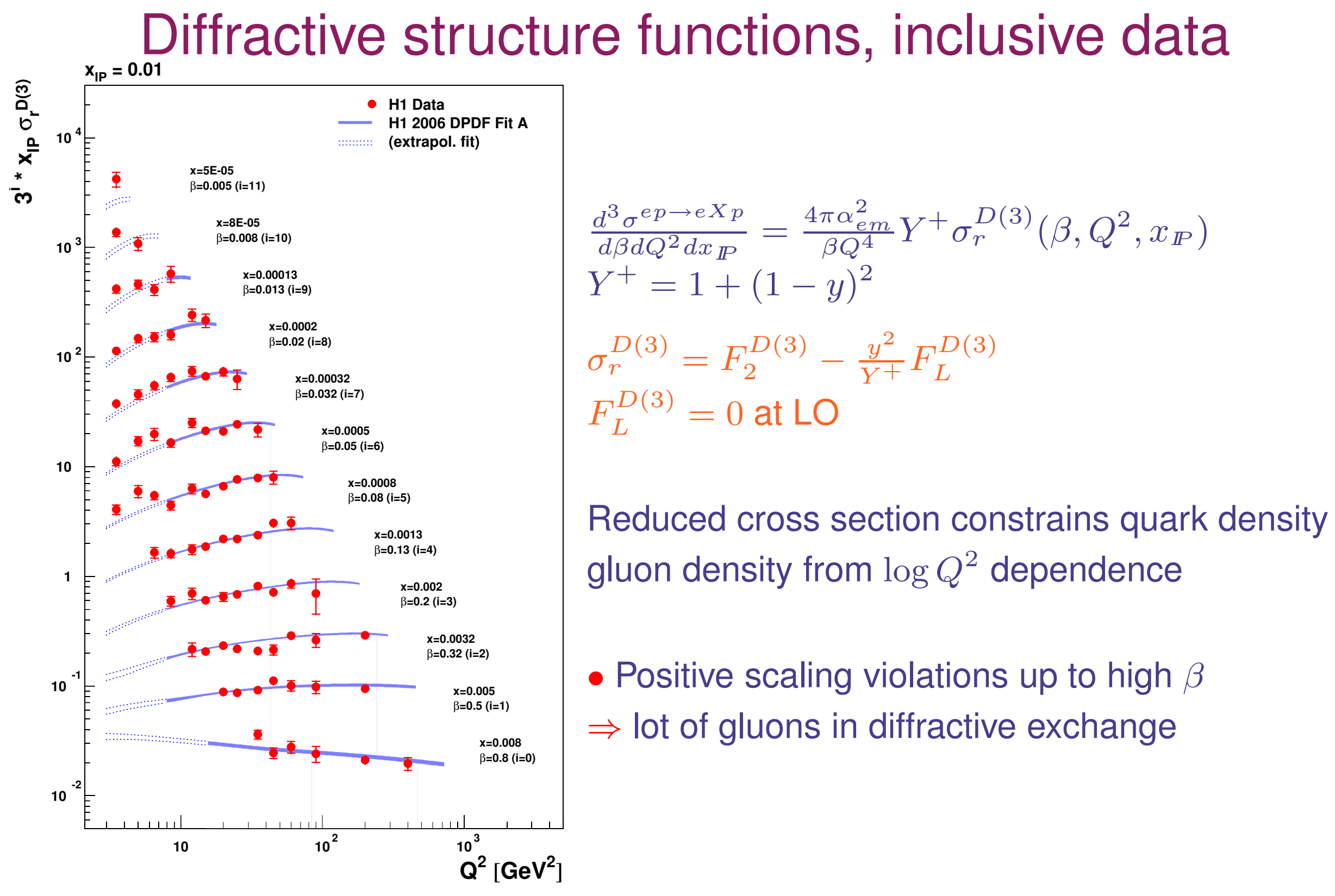




\section{Diffractive PDFs from inclusive data}

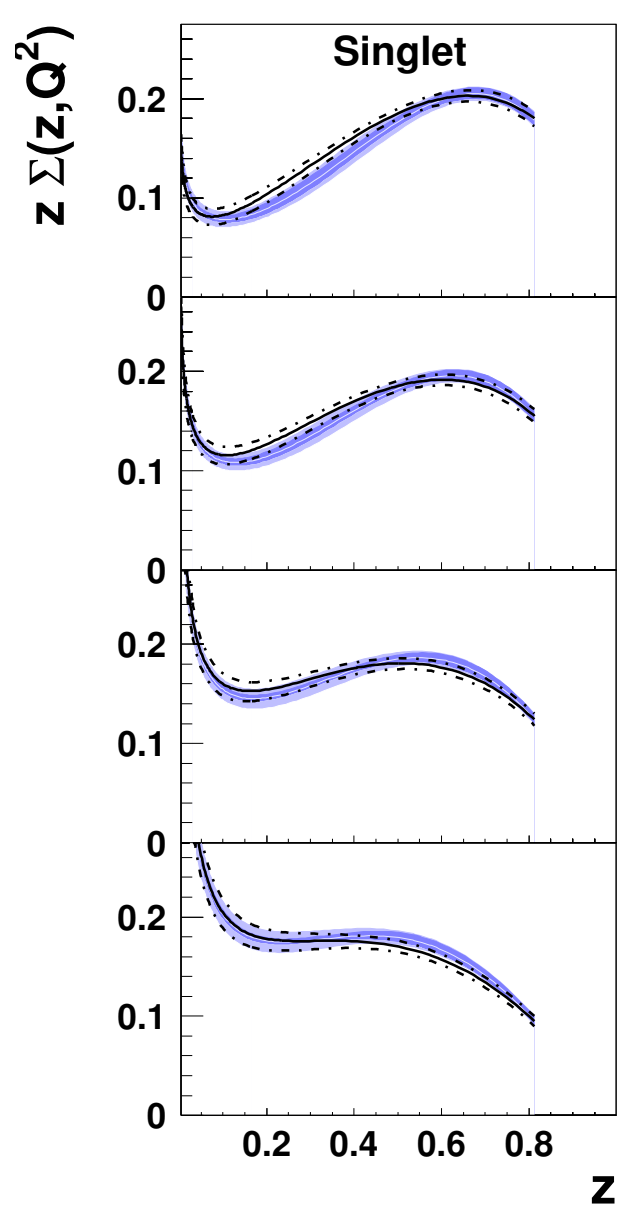

H1 2006 DPDF Fit A (exp. error) (exp.+theor. error)
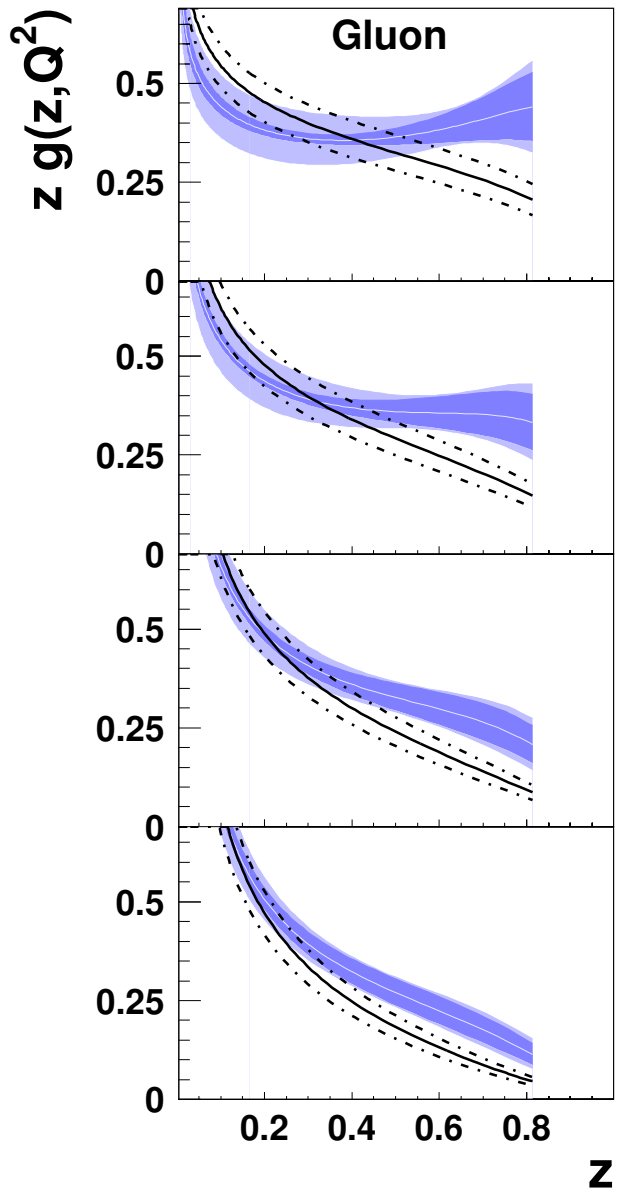

$\mathbf{z}$
$\mathrm{Q}^{2}$

- Additional assumption: proton vertex factorisation

8.5

$$
\frac{d^{2} F_{2}^{D}\left(x_{\mathbb{P}}, t, x, Q^{2}\right)}{d x_{\mathbb{P}} d t}=f_{\mathbb{P} / p}\left(x_{\mathbb{P}}, t\right) F_{2}^{\mathbb{P}}\left(\beta, Q^{2}\right)
$$

- NLO DGLAP fits:

20

- parametrise Flavour Singlet $(q, \bar{q})$ and Gluons at $Q^{2} \simeq 3 \mathrm{GeV}^{2}$

- evolve with NLO DGLAP and fit

90

-Different parametrisations:

800

H1 2006 fit A: $z g\left(z, Q_{0}^{2}\right)=A(1-z)^{B}$

H1 2006 fit B: $z g\left(z, Q_{0}^{2}\right)=A(1-z)$

but also Martin Ryskin Watt,

ZEUS fit LPS + charm ...

- H1 2006 DPDF Fit B

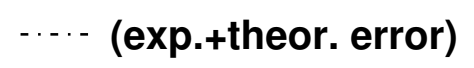

$\mathrm{Z}=$ fractional momentum of parton participating in hard scattering 


\section{Diffractive PDFs from inclusive data}

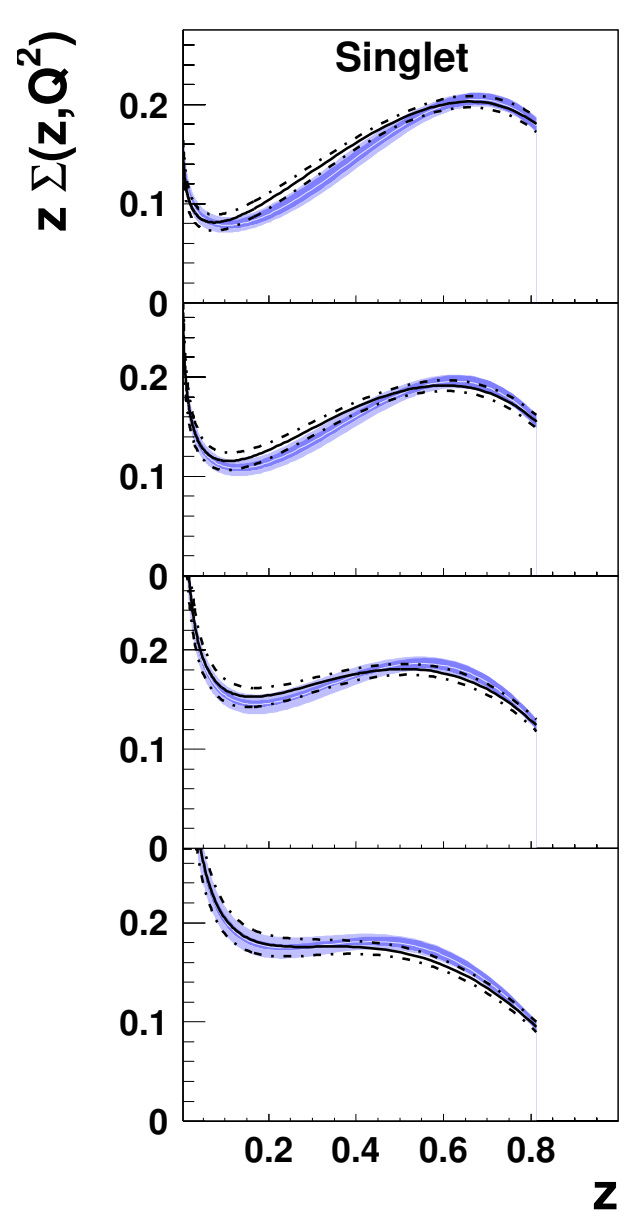

H1 2006 DPDF Fit A (exp. error)

(exp.+theor. error)
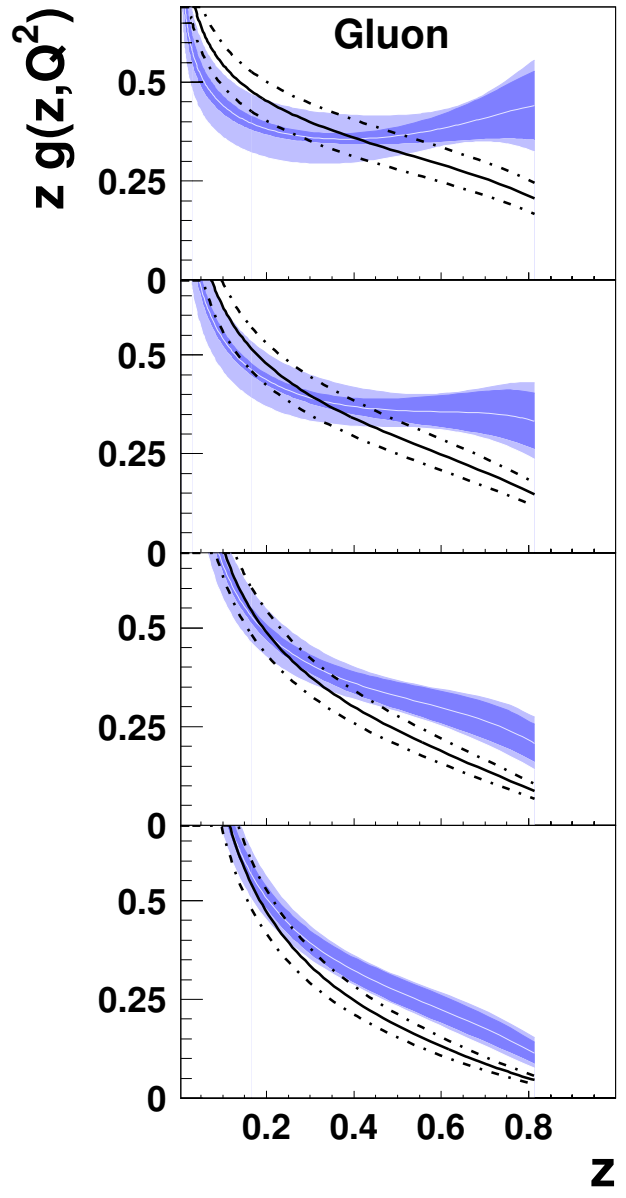

- H1 2006 DPDF Fit B

..... (exp.+theor. error)
$\mathrm{Q}^{2}\left[\mathrm{GeV}^{2}\right]$

8.5

- Well constrained singlet

- Gluon dominated, 75\% of exchanged momentum carried by gluons. Gluons 20 weakly constrained, exp. at high values of $\mathbf{z}=$ fractional momentum of parton participating in hard scattering

- Low z: evolution driven by $g \rightarrow q \bar{q}$

800 strong sensitivity to gluons

- High z: $q \rightarrow q g$ contribution to evolution dominant, relative error on derivative grows 


\section{Factorisation tests: $\mathrm{D}^{*}$ in DIS}

- Use diffractive PDFs from NLO DGLAP fits

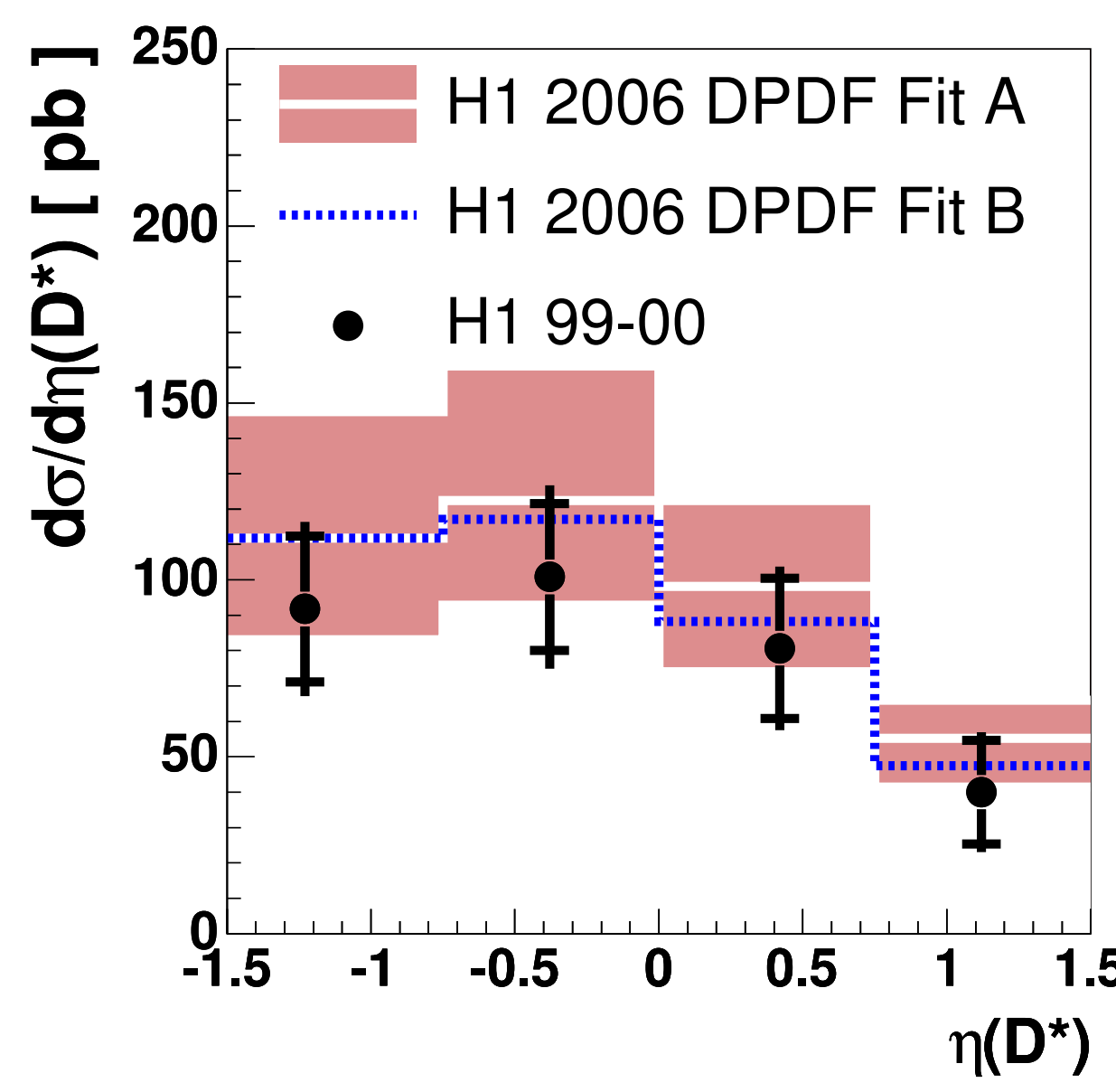
of $F_{2}^{D}$ to predict rate of charm production

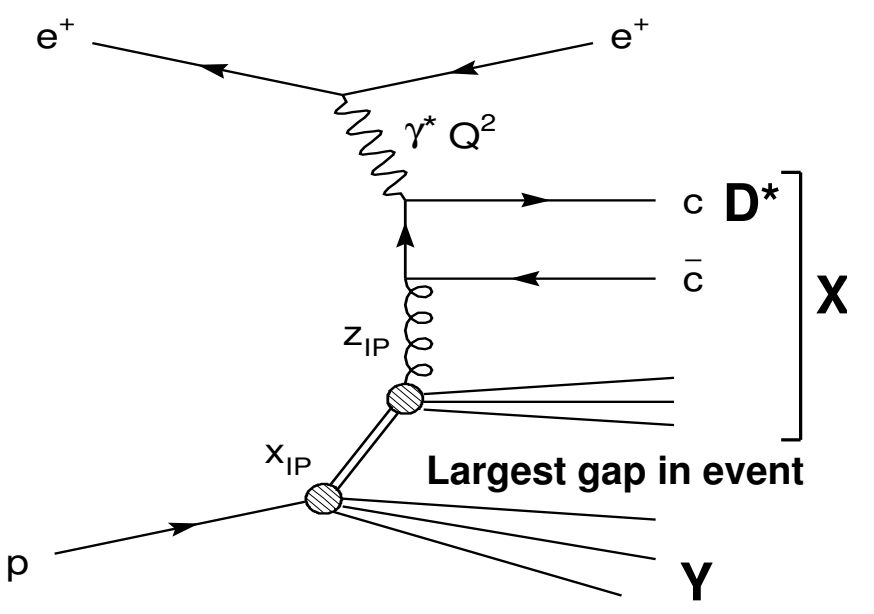

Kinematic range:

$2<Q^{2}<100 \mathrm{GeV}^{2}, p_{T}\left(D^{*}\right)>2 \mathrm{GeV}$, $\left|\eta\left(D^{*}\right)\right|<1.5,0.05<y<0.7$ 150 diffractive $D^{*}$

- NLO calc. + diffractive PDFs describe data

$\Rightarrow$ Within uncertainties, QCD factorisation works 


\section{ZEUS}
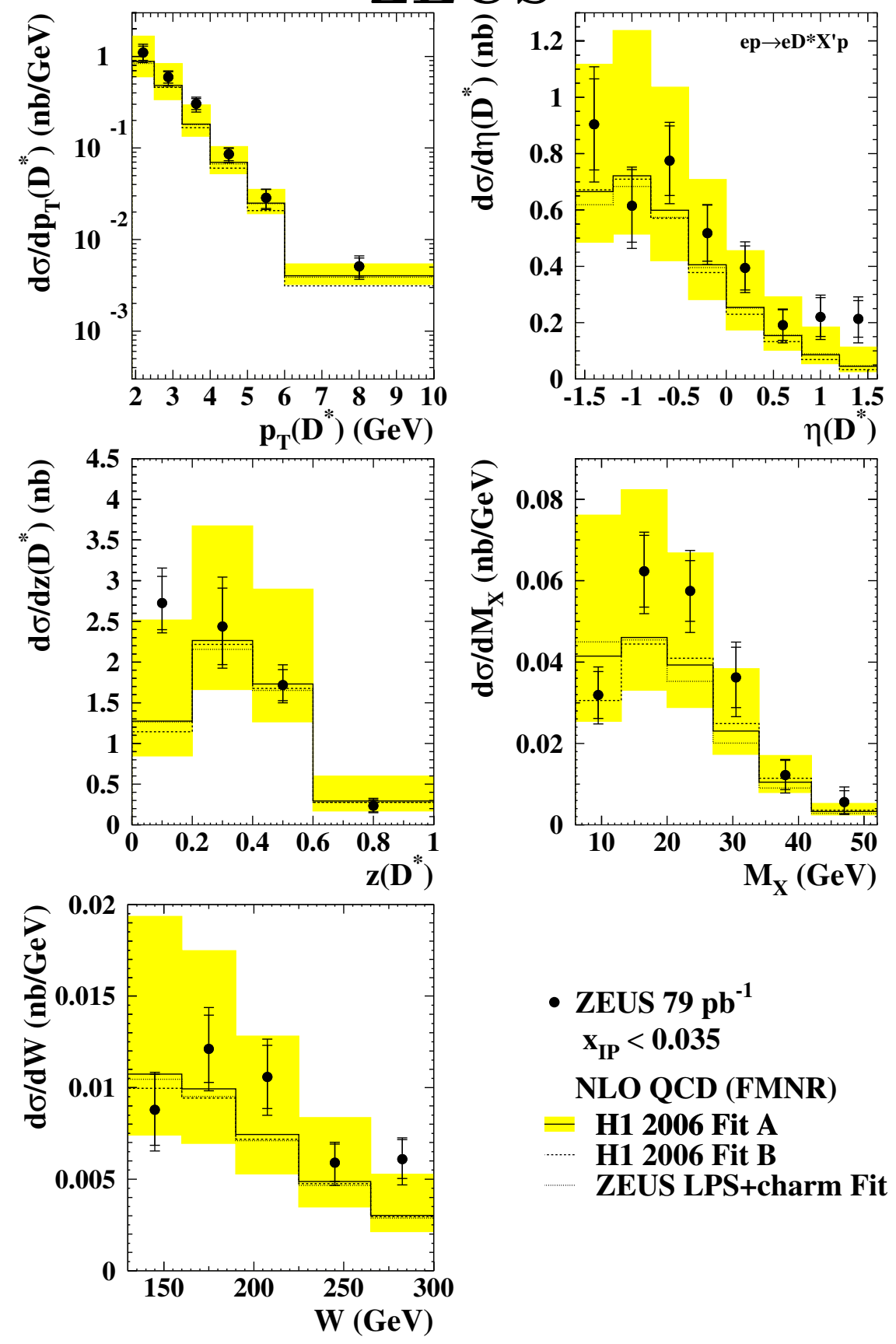

- ZEUS 79 pb $^{-1}$ $\mathrm{x}_{\mathrm{IP}}<\mathbf{0 . 0 3 5}$ NLO QCD (FMNR)

= H1 2006 Fit A H1 2006 Fit B

ZEUS LPS+charm Fit

\section{Factorisation tests: $\mathbf{D}^{*}$ in $\gamma p$}

Kinematic range:

$130<W<300 \mathrm{GeV}, p_{T}\left(D^{*}\right)>1.9 \mathrm{GeV}$, $\left|\eta\left(D^{*}\right)\right|<1.6$

$12500 D^{*}, 200$ diffractive $D^{*}$

In the kin. region of the measurement, $D^{*}$ production mainly produced in direct photon processes $\rightarrow$ no suppression expected

- NLO calc. + diffractive PDFs describe data $\Rightarrow$ No evidence of factorisation breaking 


\section{ZEUS}
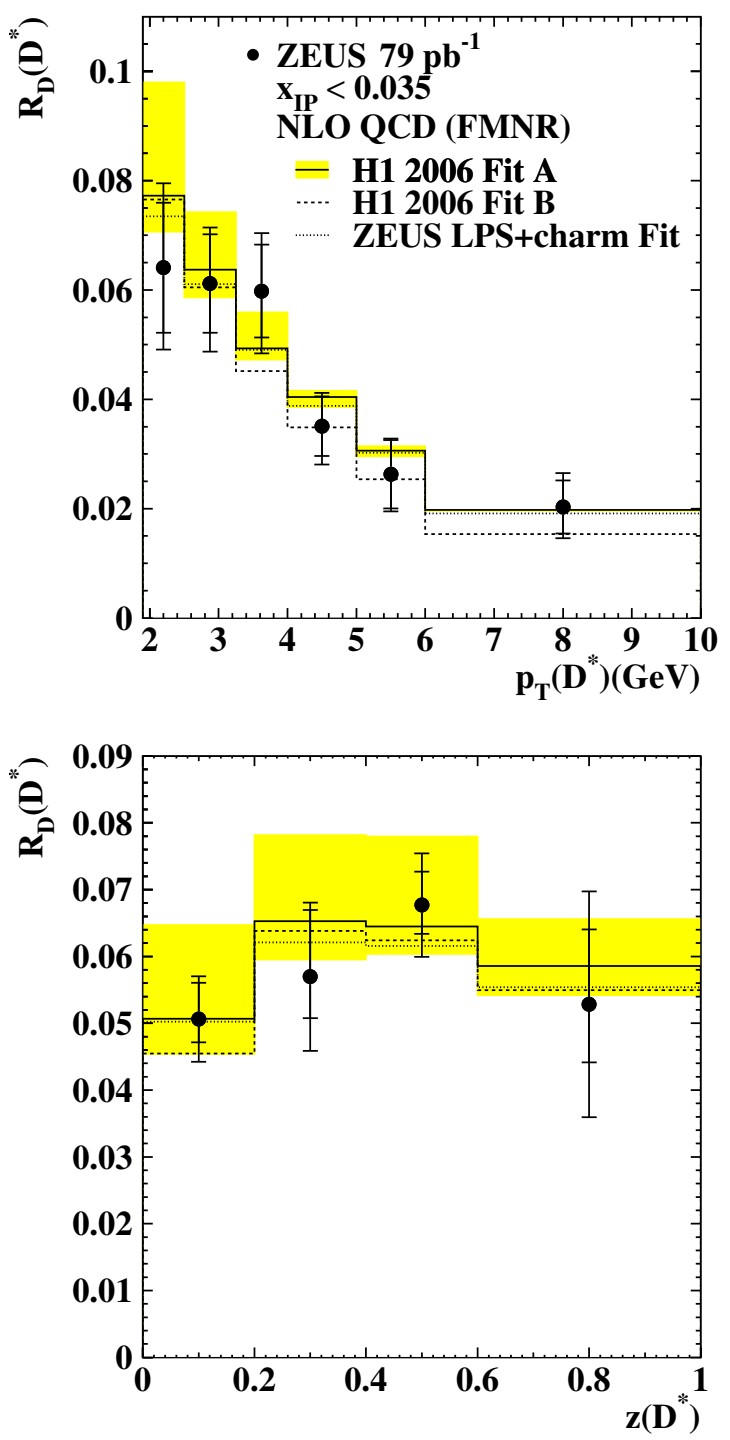
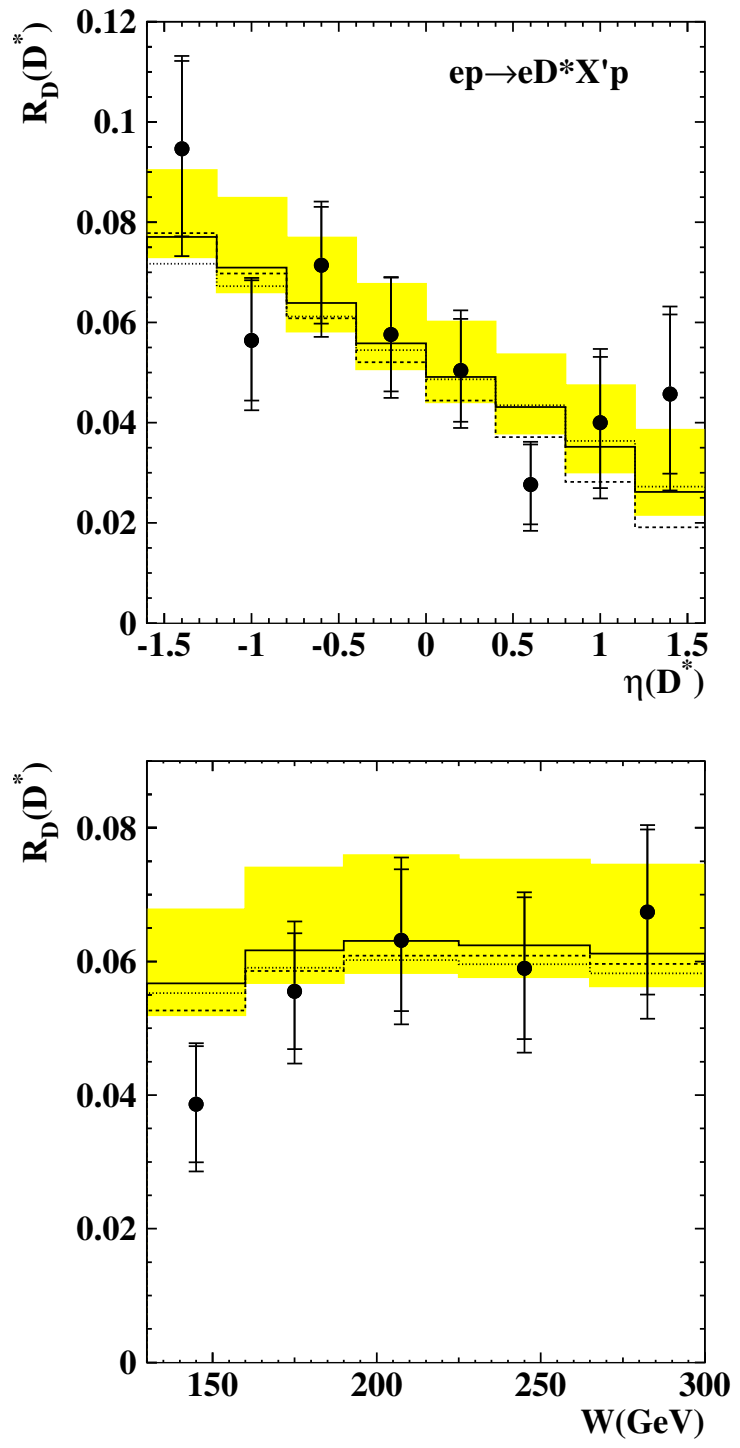

\section{Factorisation tests: $\mathrm{D}^{*}$ in $\gamma p$}

- Ratio diffractive/inclusive

$R_{D}=5.7 \pm 0.5 \%$

- Ratio from NLO calculations:

$\mathrm{H} 1$ fit $2006 \mathrm{~B} \Rightarrow 5.7 \%$

ZEUS fit LPS + charm: $\Rightarrow 5.8 \%$

$\Rightarrow$ No evidence of factorisation breaking 


\section{Factorisation tests: dijet in DIS ZEUS}
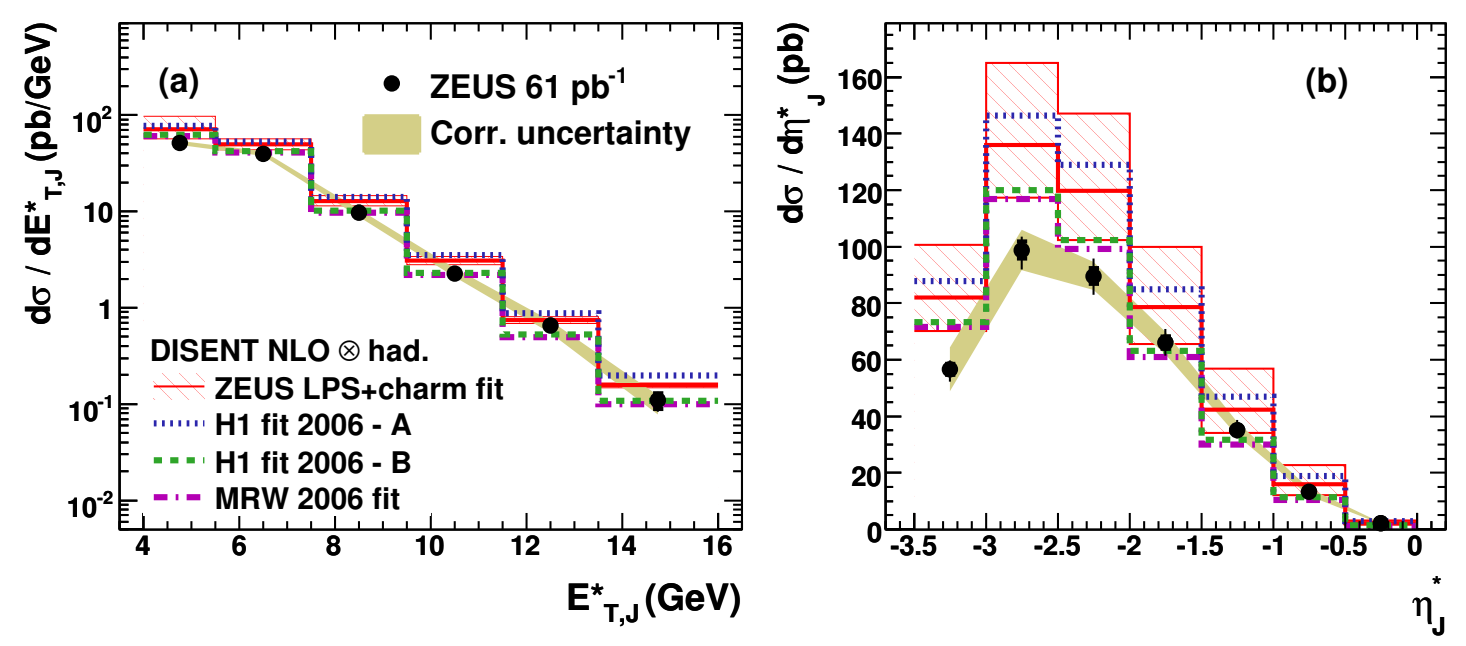

Kinematic range:
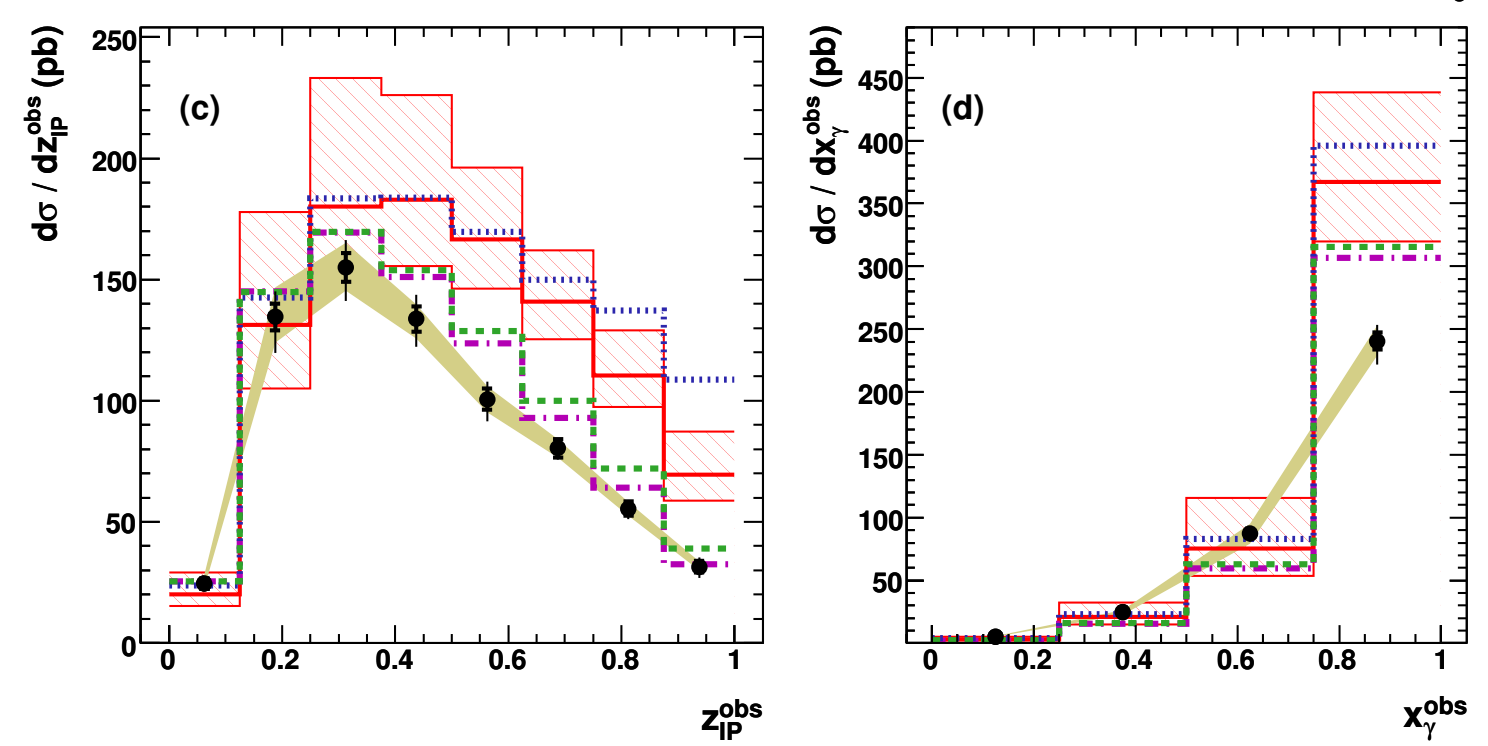

$5<Q^{2}<100 \mathrm{GeV}^{2}$,

$E_{\text {Tjet1(jet } 2)}>$ 4.(5.) GeV

- NLO predictions using diffractive PDFs describes data within theoretical uncertainties

- Agreement depends on kinematic region $(0-25 \%)$

- Large discrepancy between different diffractive PDFs, best agreement for H1 2006 fit B and Martin Ryskin Watt 


\section{Factorisation tests: dijet in DIS ZEUS}
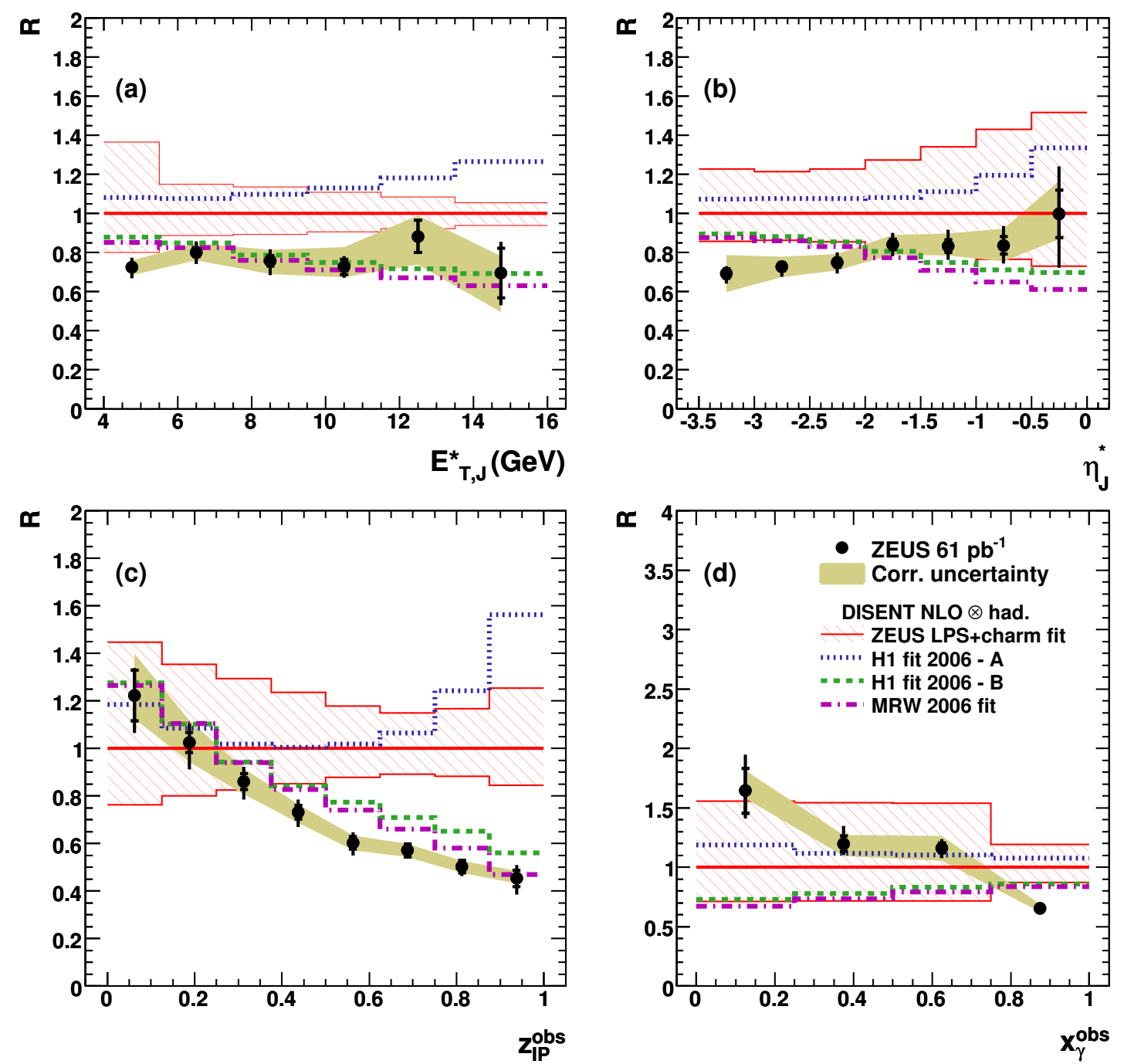

- $\mathrm{R}=$ data/NLO (LPS + charm)

- Discrepancy at large z, new fits:

- H1 2007 Jets DPDF

...... H1 2006 DPDF fit B

…….. H1 2006 DPDF fit A

...... Martin, Ryskin, Watt 2006
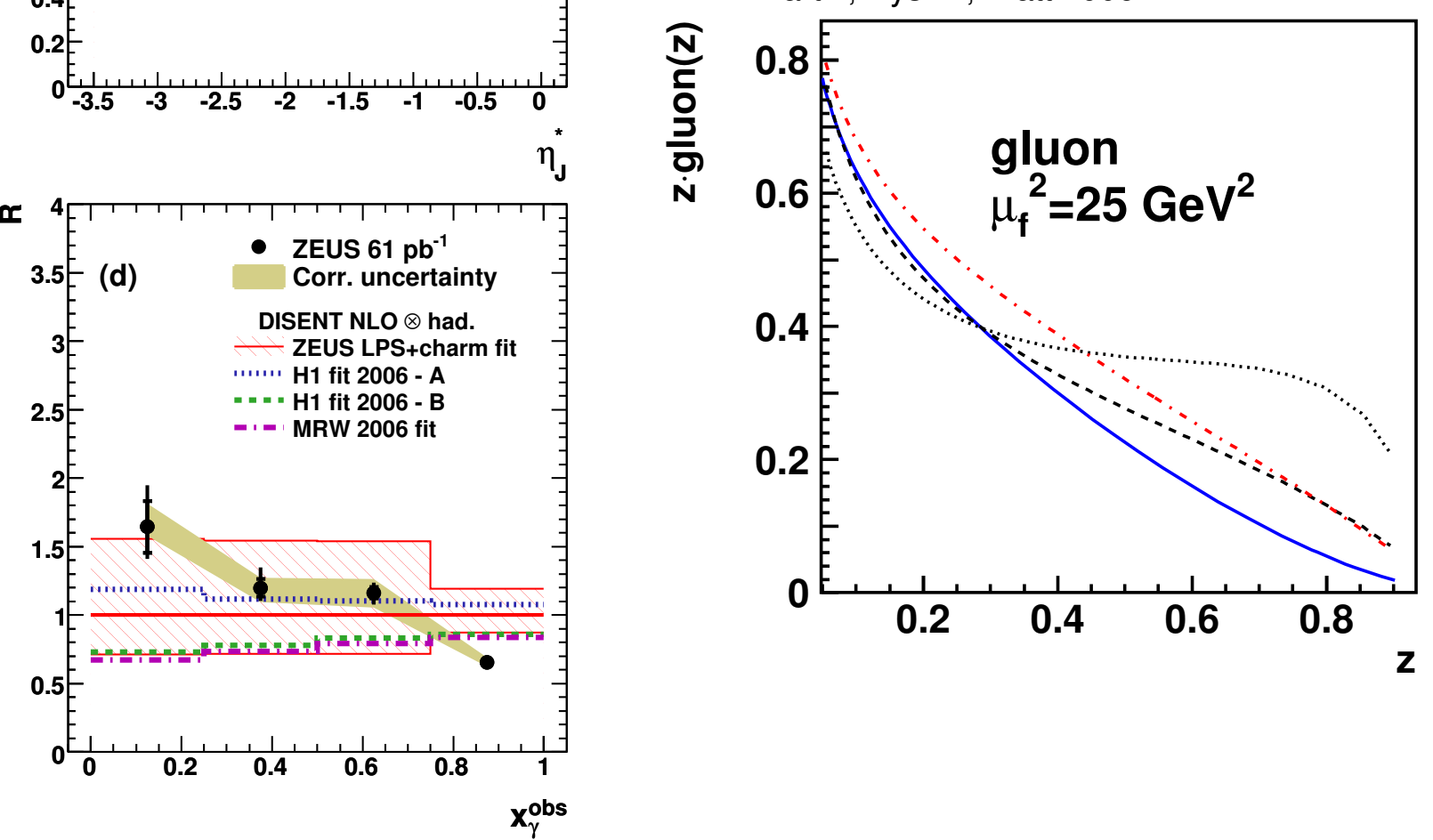


\section{Combined fit to inclusive and dijet DIS data}

\section{H1 data}

\section{- H1 2007 Jets DPDF}

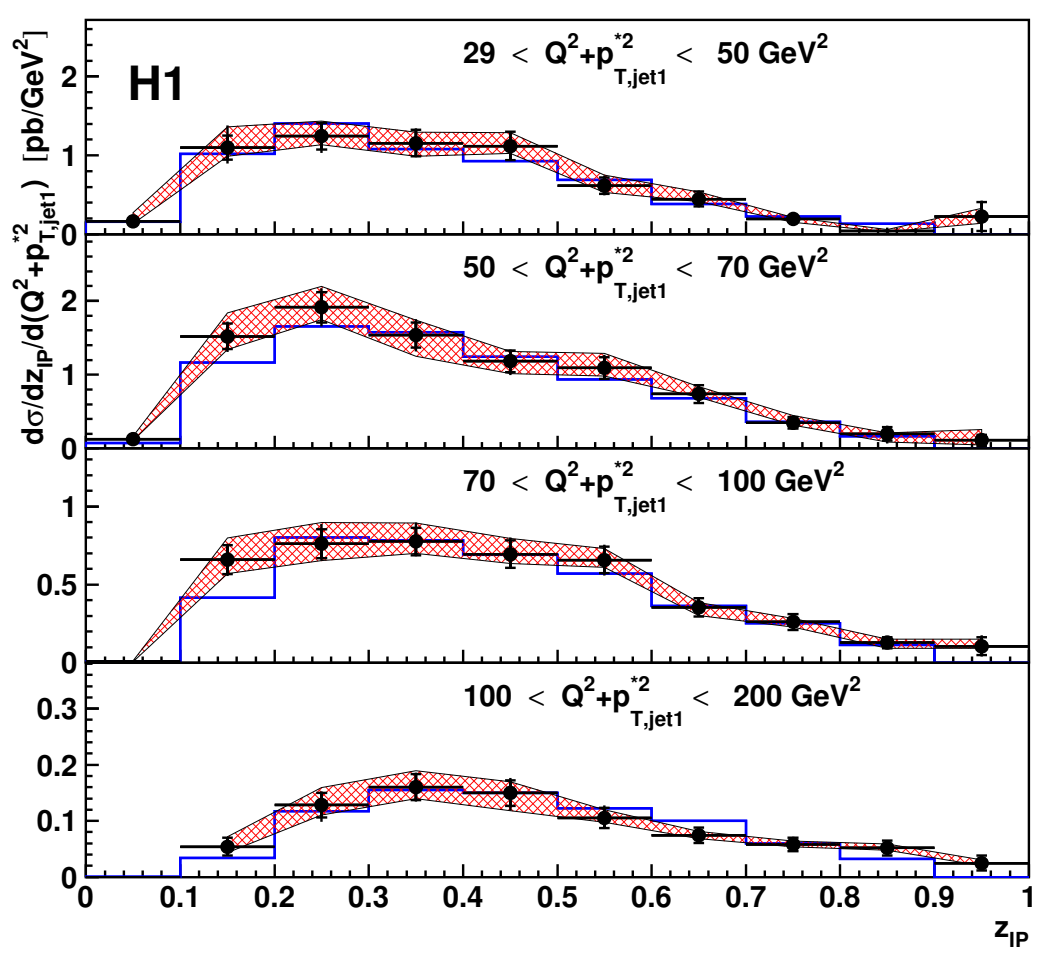

$x_{I P}=0.01$

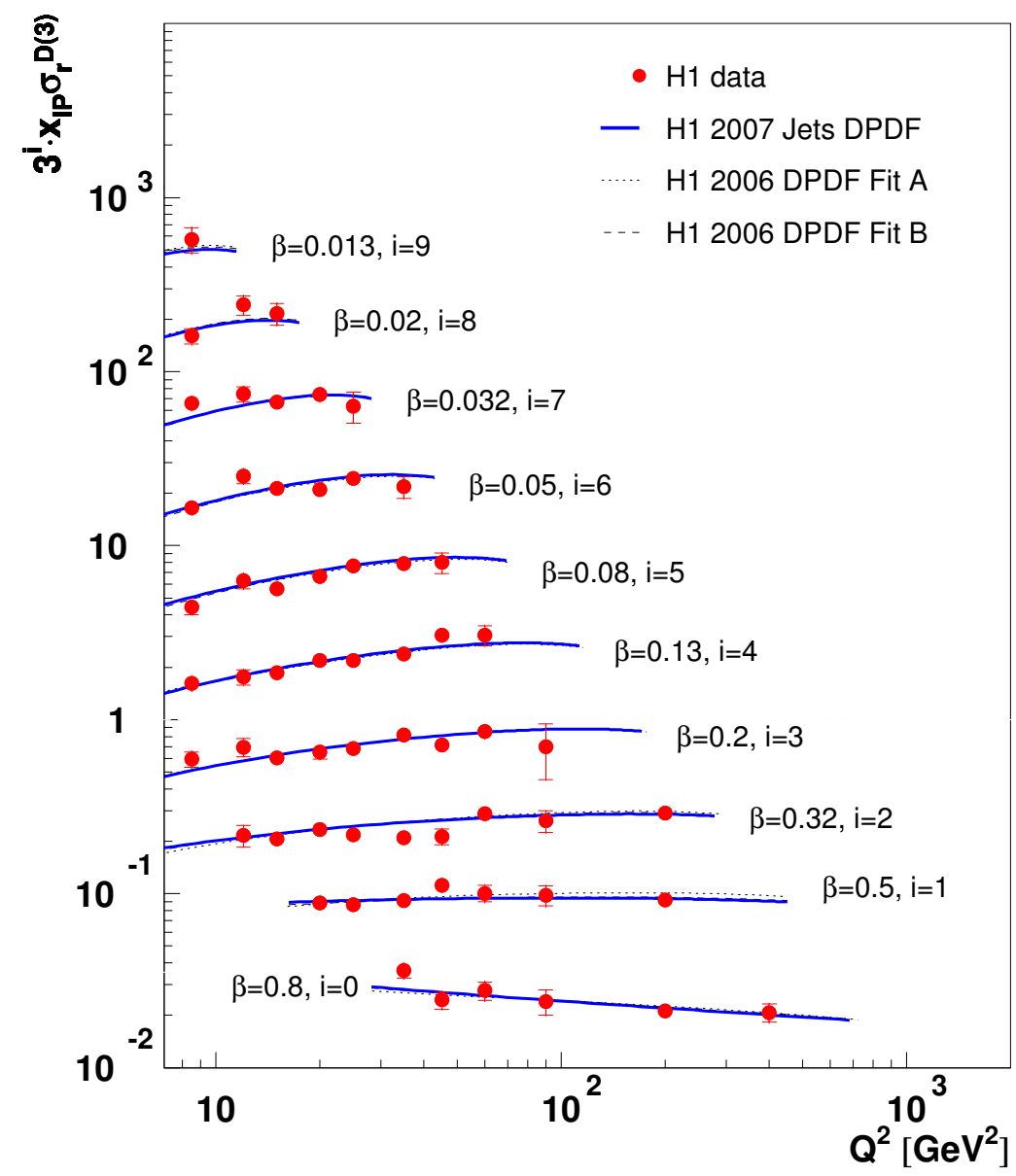

- Combined fit constrains quark and gluon densities over a wide range $0.05<z<0.9$

$\Rightarrow$ Uncertainty on gluon density reduced 


\section{Factorisation tests in $\gamma p$}

Double ratio of (data/NLO $)^{\gamma p} /(\text { data/NLO })^{D I S}$, to cancel diffractive PDF uncertainty

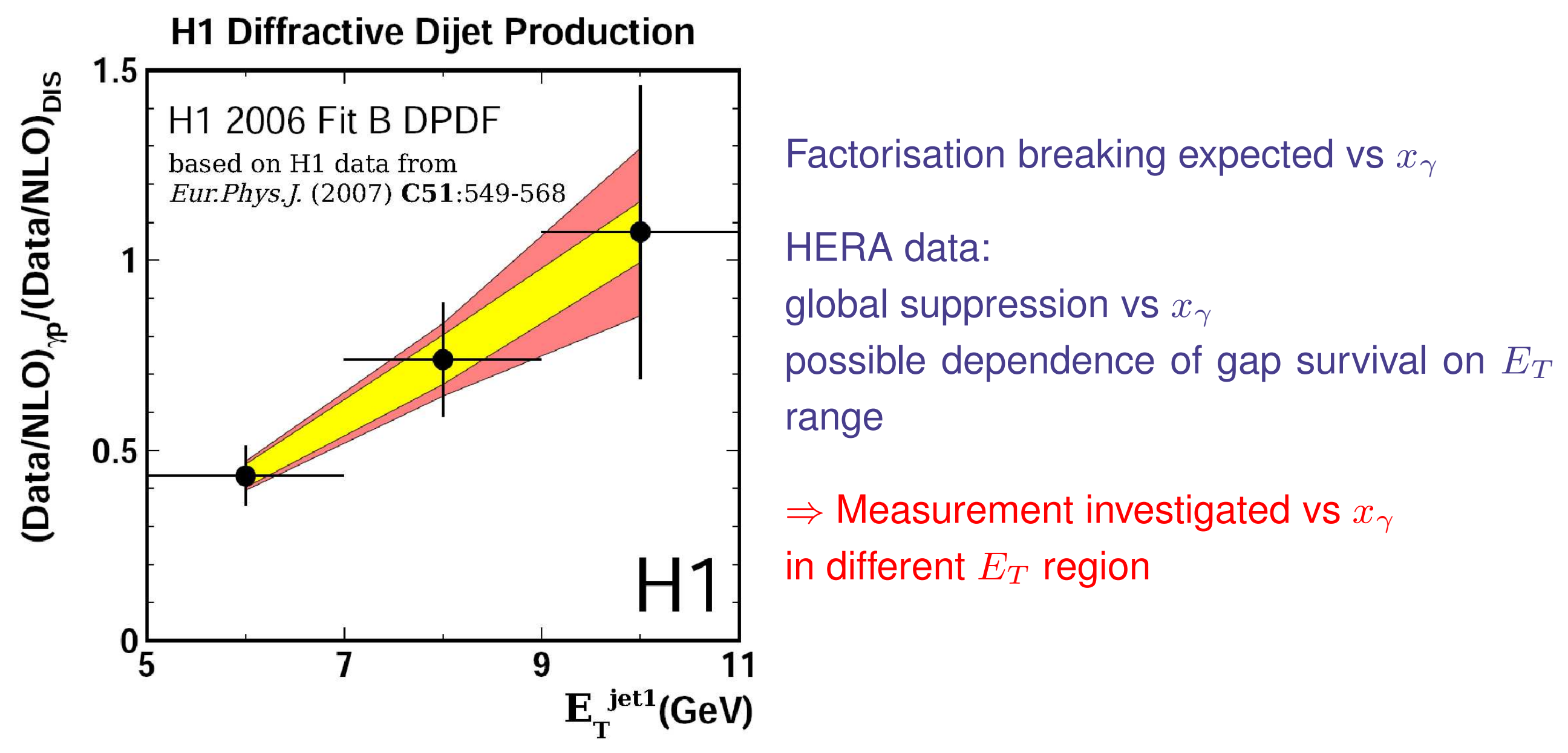




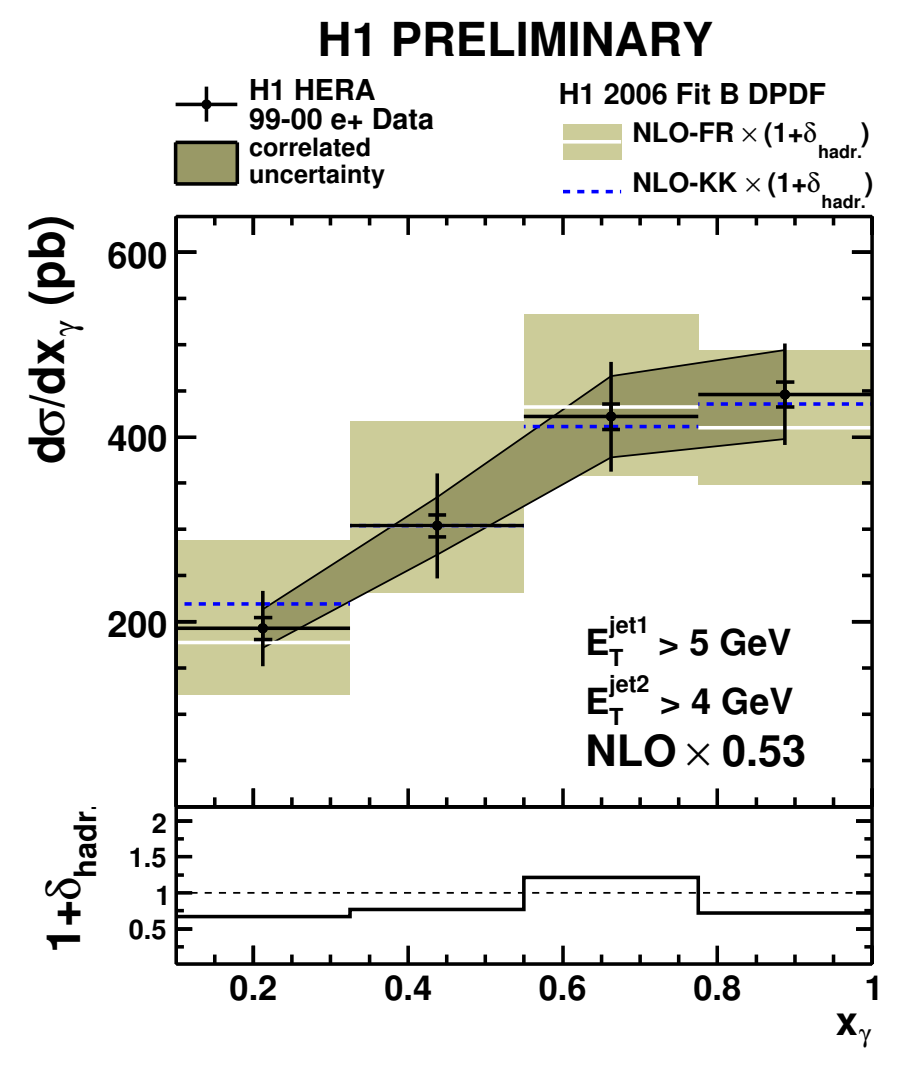

H1 PRELIMINARY

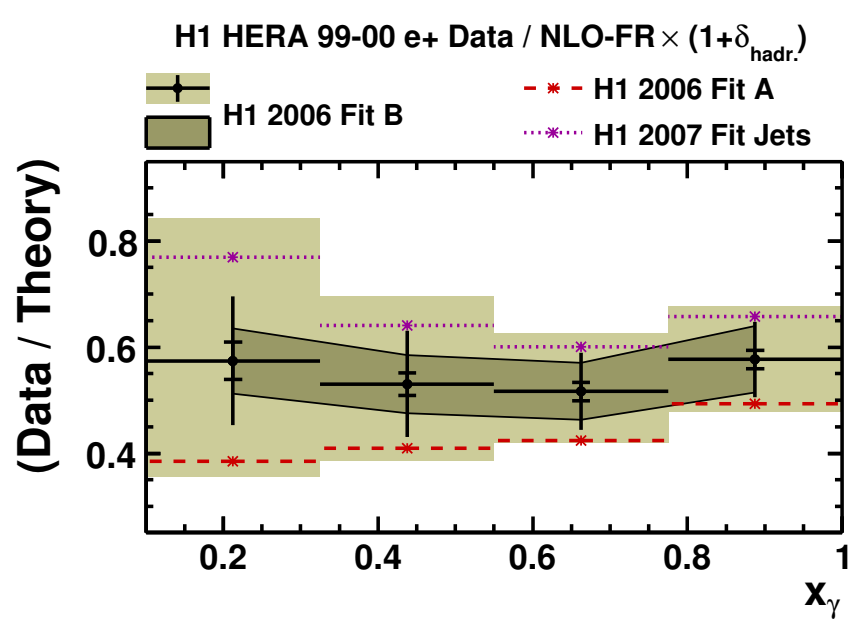

\section{Factorisation tests in $\gamma p$}

-Low $E_{T}$

$E_{\text {Tjet1(jet2) }}>$ 5.(4.) GeV

$-1 .<\eta^{j e t}<2$

$0.3<y<0.65$

- Prediction extrapolated at high z, beyond the range of the diffractive PDF

$\Rightarrow$ No evidence of suppression of resolved contribution only

$\Rightarrow$ Global suppression data $/ \mathrm{NLO}(\mathrm{H} 1$ fit $\mathrm{B})=0.54 \pm 0.10$ (syst) \pm 0.14 (scale) data $/ \mathrm{NLO}(\mathrm{H} 1$ fit jets $)=0.65 \pm 0.11$ (syst) 


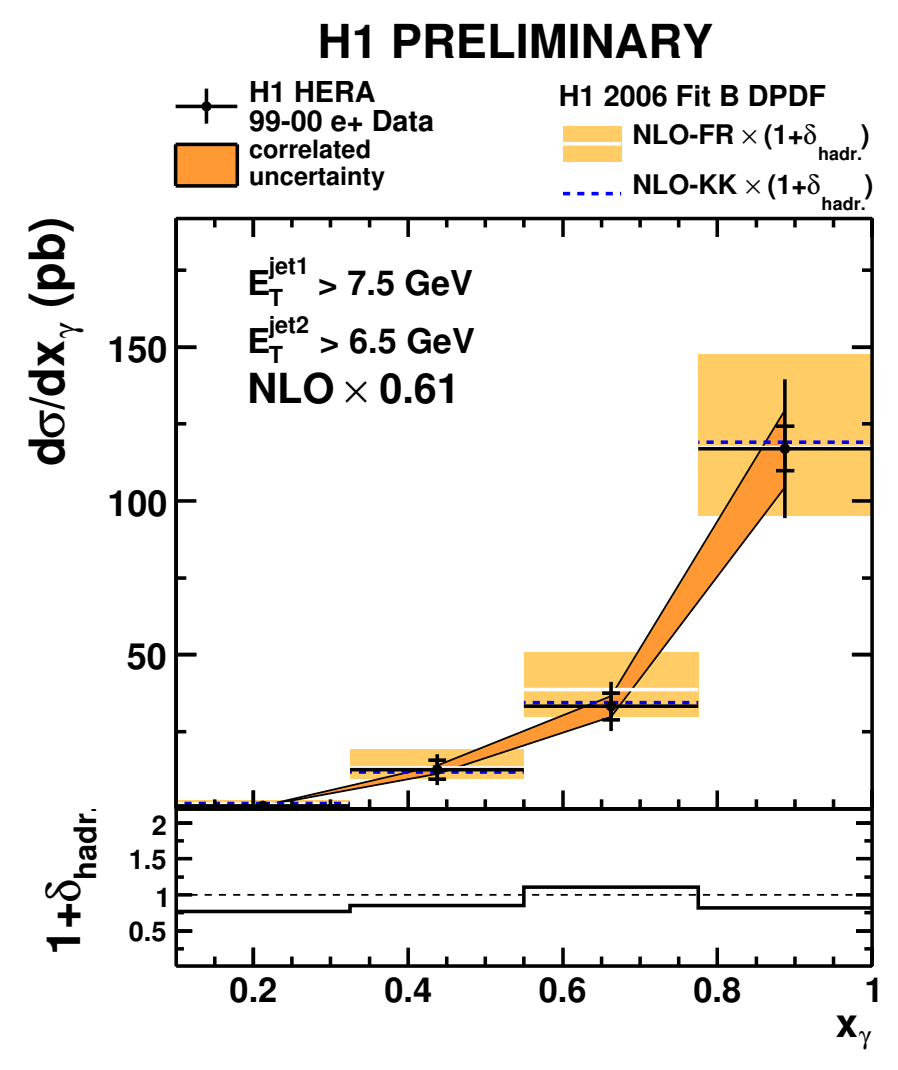

H1 PRELIMINARY

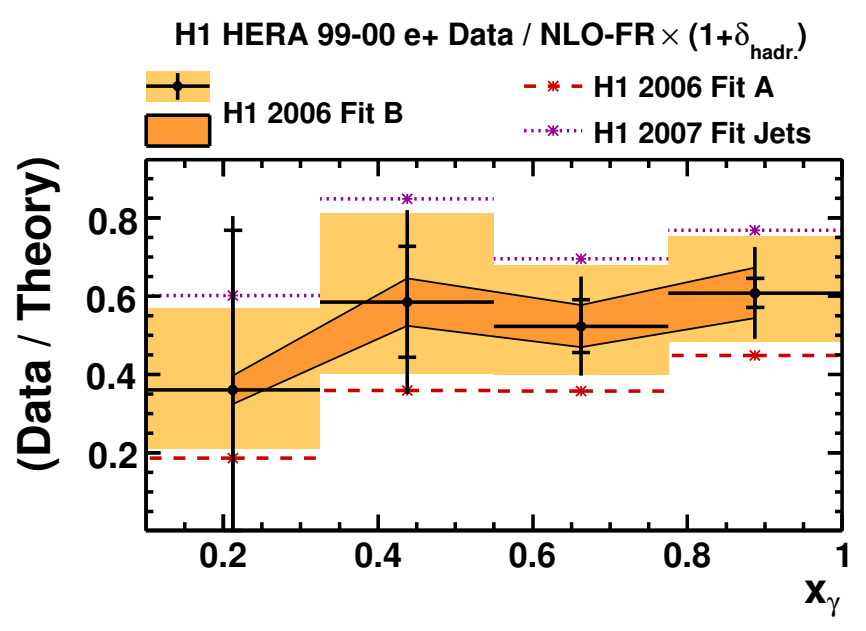

\section{Factorisation tests in $\gamma p$}

•High $E_{T}$

$E_{\text {Tjet1(jet2) }}>7.5(6.5) \mathrm{GeV}$

$-1.5<\eta^{j e t}<1.5$

$0.3<y<0.65$

$\Rightarrow$ No evidence of suppression of resolved contribution only

$\Rightarrow$ Global suppression

data / NLO(H1 fit B $)=0.61 \pm 0.13$ (syst) \pm 0.15 (scale)

data $/ \mathrm{NLO}(\mathrm{H} 1$ fit jets $)=0.79 \pm 0.16($ syst $)$ 


\section{ZEUS}

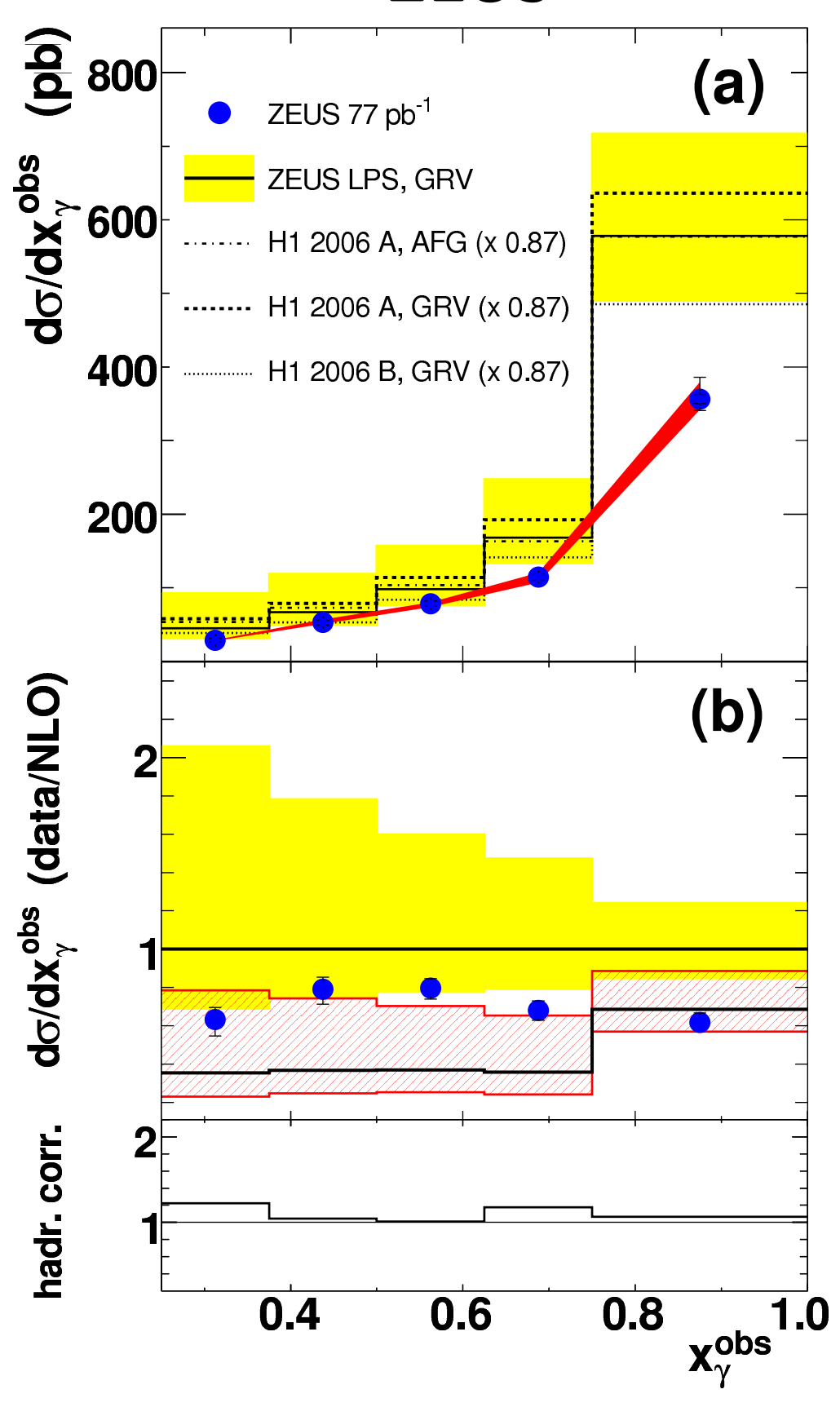

-High $E_{T}$

$E_{T j e t 1(j e t 2)}>7.5(6.5) \mathrm{GeV}$

$-1.5<\eta^{j e t}<1.5$

$0.2<y<0.85$

$\Rightarrow$ Large theoretical uncertainty

$\Rightarrow$ No suppression of resolved contribution only

$\Rightarrow$ Global suppression

data / NLO(ZEUS LPS + charm $) \simeq 0.6$

data $/ \mathrm{NLO}(\mathrm{H} 1$ fit $\mathrm{B}) \simeq 0.9$

$\rightarrow$ data compatible with no or small suppression, depending on diffractive PDFs

$\rightarrow$ Indication of possible suppression at small $E_{T}$ 


\section{Uncertainties for predictions}

- Large scale dependence

- higher QCD order important

- Flavour scheme dependence

DIS, PHP

- dPDF fitted using 3-flavour FFNS + massive c,b

- dijets calculated with all flavours massless ZM-VFNS

- Gluon content of the Pomeron

- poorly constrained at high z

- Proton dissociation correction factor

- $10 \%$ uncertainty when using H1 dPDF to ZEUS

PHP

- Factorisation scheme dependence for the photon

- quark/gluon content of the photon

$\Rightarrow$ up to $\sim 30 \%$ uncertainty 


\section{Another test of factorisation breaking:}

\section{Dijet photoproduction with a leading neutron}




\section{Dijet photoproduction with a leading neutron}

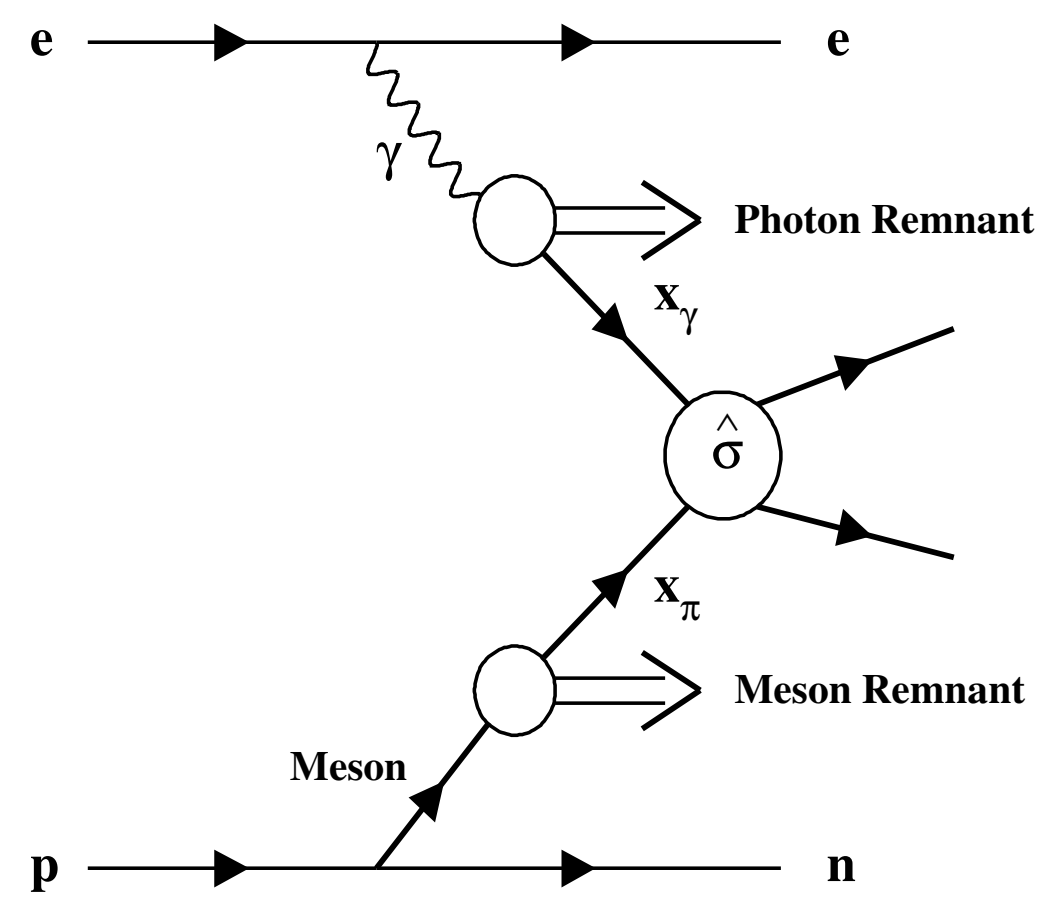

- Factorisation breaking expected in dijet photoproduction with a leading neutron $\gamma p \rightarrow j j n X$; • events whith a non-diffractive exchange (pion exchange)

- Soft rescattering expected between $\gamma$ remnant and $n$

- NLO predictions (non-perturbative pion flux factor) normalised to DIS $e p \rightarrow$ ejjXn data and compared to photoproduction data, looking for suppression 


\section{Dijet photoproduction with a leading neutron}

\section{ZEUS}

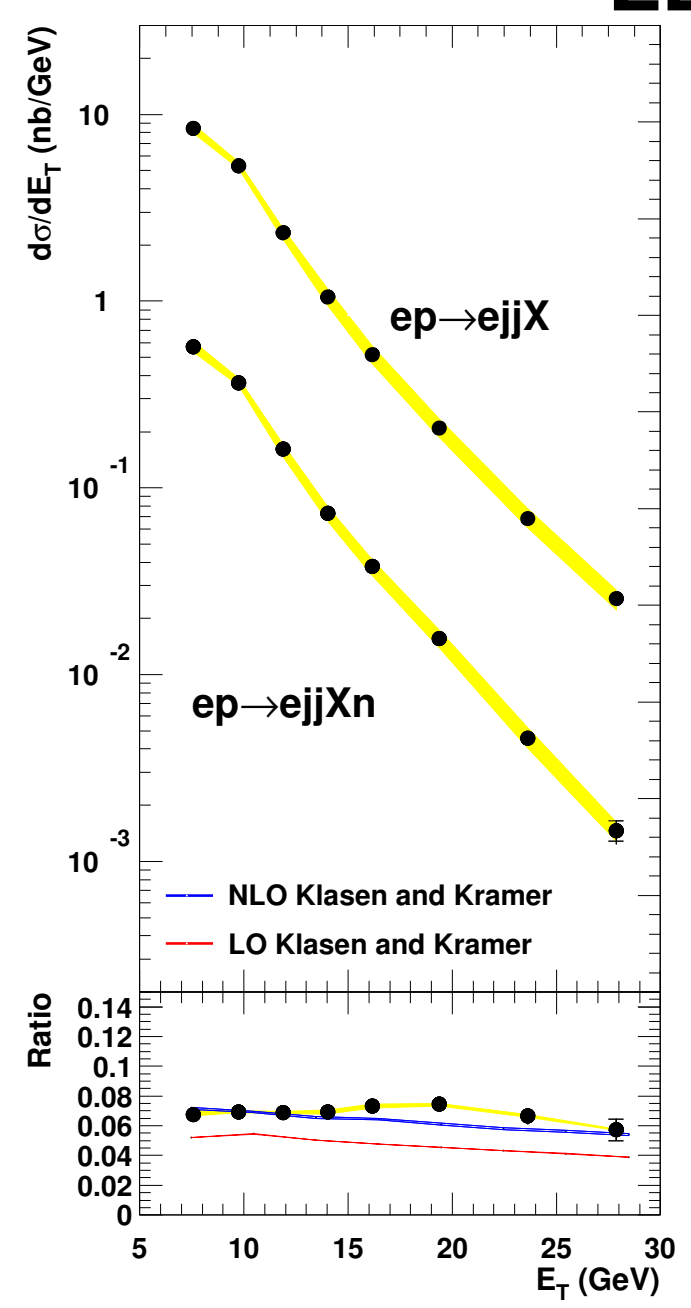

kinematic range:

$E_{\text {Tjet1(jet2) }}>7.5(6.5) \mathrm{GeV}$

$-1.5<\eta^{j e t}<2.5$

$130<W<280 \mathrm{GeV}$

$E_{n}>184 \mathrm{GeV}$

- Ratio $(e p \rightarrow e j j X n) /(e p$

ejjX) compared to NLO (hadronisation corr. cancel in ratio)

$\Rightarrow$ NLO predictions describe data

- data consistent with factorisation of lepton and hadron vertices and onepion-exchange model

$\Rightarrow$ no sign of factorisation breaking 


\section{QCD factorisation tests summary}

Factorisation studied within QCD framework:

- New diffractive PDFs available:

- inclusion of dijet data in the fits provides a better constraint on gluon density

Diffractive charm in PHP and DIS:

- within low statistics and large NLO uncertainties no hint of factorisation breaking observed

Diffractive dijets:

- diffractive dijet production measured in wide range of photon virtualities (0-100 $\left.\mathrm{GeV}^{2}\right)$

- experimental errors much smaller than theoretical uncertainties

- NLO predictions based on dPDFs from inclusive data describes data in shape

- in PHP, data favor a global suppression of NLO QCD rather than a suppression of only the resolved component

Factorisation breaking observed at low $E_{T}$ 


\section{Conclusions}

- QCD factorisation investigated at HERA in many final states and over a wide kinematic range

- Indications of factorisation breaking observed in dijets photoproduction at low $E_{T}$ (where resolved processes dominate), but not clearly vs $x_{\gamma}$

- Exp: cross-section ratios can be useful, i.e. ratio diffractive/inclusive production vs $E_{T}, x_{\gamma}$

- Better QCD prediction needed (consistent flavours/masses treatement, showering ...) to quantify the suppression 